\title{
Statistical analysis of Bragg reflectors*
}

\author{
J. B. Shellan, ${ }^{\dagger}$ P. Agmon, P. Yeh, and A. Yariv \\ California Institute of Technology, Pasadena, California 91125
}

(Received 12 September 1977)

\begin{abstract}
The effects on reflectivity of a statistical variation in the thickness of layers in a multilayered Bragg reflector are studied. Analytic expressions are obtained for $\langle\rho\rangle$ and $\left\langle\rho \rho^{*}\right\rangle$, the expected value of the reflection and reflectivity coefficients as a function of $\sigma$, the standard deviation in layer thickness. These expressions are then compared with values obtained using a computer routine which "builds" a reflector with the desired parameters and $\sigma$ value, and then calculates the reflection. The results of the computer experiment are presented in the form of $p\left(\rho \rho^{*}\right)$, the probability distribution function of a statistical Bragg reflector. Finally, simple phenomenological expressions are presented for the reflectivity probability distribution.
\end{abstract}

\section{INTRODUCTION}

Extensive studies have been made of the reflection of light from ideal periodic multilayered media. ${ }^{1,2}$ Among the many uses of such structures are coatings for both high reflection and antireflection. Other proposals involve the use of these structures for phase matching in nonlinear optical applications $^{3-5}$ and for obtaining optical birefringence in stratified media composed of isotropic or cubic materials. 6,7

In practice, however, it is not possible to fabricate perfect structures, and to date the standard deviation in layer thicknesses of commercially made mirrors is typically $2 \%$ when monitored optically, and even greater when measured mechanically. 8,9 Great precision in layer thickness can be achieved by using new techniques such as molecular beam epitaxy, but these techniques are also costlier than the standard electron beam evaporation.

Although there is ample literature on periodic structures, the study of aperiodic structures has been rather limited. ${ }^{10-12}$ The primary effect of a slight aperiodicity is to decrease the amplitude and broaden the width of the reflectivity spectrum. It is the purpose of this paper to study the effect on reflectivity of a random fluctuation in layer thickness about an ideal thickness.

The case of a low reflectivity structure is easily handled using the undepleted incident wave approximation. Next a

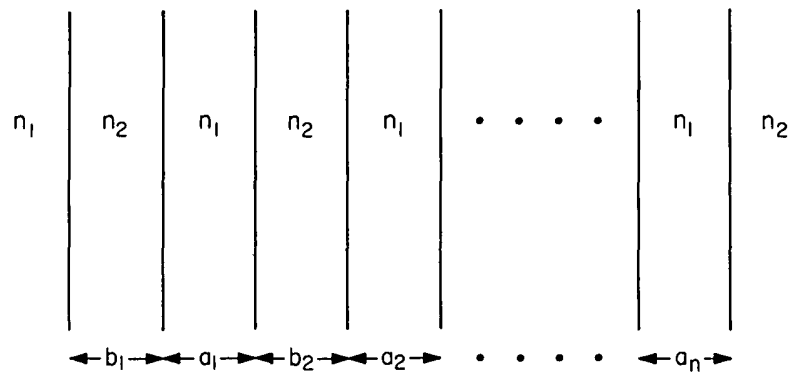

FIG. 1. Geometry of reflection with $n$ cells used in low-reflectivity case.

perturbation solution to the coupled mode equations is presented which gives results for arbitrarily large reflectances. Finally, a computer study is presented which uses the formalism of the matrix and translation operator developed by Yeh, Yariv, and Hong ${ }^{2}$ to predict the expectation value of $\rho$ and $|\rho|^{2}$ as a function of $\sigma$ as well as $p\left(|\rho|^{2}\right)$, the probability of manufacturing a sample of given reflection. An analytic expression is then presented for $p\left(|\rho|^{2}\right)$ which agrees well with the results from the computer experiment.

\section{LOW-REFLECTIVITY LIMIT}

We start by calculating the reflectivity of a mirror with $N$ cells in the limit of low reflectance. Assuming a constant incident wave of unit amplitude we obtain for the reflected wave

$$
\begin{array}{r}
\rho=r_{1}\left(1+\exp \left[2 i\left(k_{1 x} a_{1}+k_{2 x} b_{1}\right)\right]+\exp \left\{2 i\left[k_{1 x}\left(a_{1}+a_{2}\right)+k_{2 x}\left(b_{1}+b_{2}\right)\right]\right\}+\cdots+\exp \left\{2 i \left[k_{1 x}\left(a_{1}+a_{2}+\cdots+a_{N}\right)+k_{2 x}\left(b_{1}+b_{2}+\right.\right.\right.\right. \\
-r_{1} \exp \left(2 i k_{2 x} b_{1}\right)\left(1+\exp \left[2 i\left(k_{1 x} a_{1}+k_{2 x} b_{2}\right)\right]+\cdots+\exp \left\{2 i\left[k_{1 x}\left(a_{1}+\cdots+a_{N-1}\right)+k_{2 x}\left(b_{2}+\cdots+b_{N}\right)\right]\right\}\right), \quad(1)
\end{array}
$$

where

$$
k_{i x}=(\omega / c) n_{i} \cos \theta_{i}, \quad i=1,2, \quad r_{1}=\frac{k_{1 x}-k_{2 x}}{k_{1 x}+k_{2 x}},
$$

$\omega$ is the radian frequency of light, $c$ is the velocity of light, and $n_{1}$ is the index of refraction in a layer of material 1 , and $n_{2}$ is the index in a layer of material 2. N is the number of unit cells and the number of dielectric interfaces is $2 N+1$, with $r_{1}$ representing the magnitude of the reflection from a single layer, $a_{p}$ is the thickness of the layer of index $n_{1}$ in the $p$ th cell, and $b_{p}$ is the thickness of the layer of index $n_{2}$ in the $p$ th cell (see Fig. 1).

We denote the random deviation of the layers' thickness by parameters $u_{p}, v_{p}$ defined by

$$
\begin{gathered}
a_{p}=a^{(0)}+u_{p}, \quad p=1,2, \cdots N \\
b_{p}=b^{(0)}+v_{p}, \\
a^{(0)}, b^{(0)}=\text { ideal thickness of layers, }
\end{gathered}
$$

and $u_{p}, v_{p}$ are random variables with assumed Gaussian distribution and standard deviations $\sigma_{a}$ and $\sigma_{b}$.

In the process of taking the ensemble average of $r$ we use the following theorem: If $G$ is a random Gaussian variable with average value zero and standard deviation $\sigma_{G}$, then the ensemble average of $e^{i G} \equiv\left\langle e^{i G}\right\rangle=e^{-1 / 2 \sigma_{G}^{2}}$. This can easily be shown by expanding $e^{i G}$ in a Taylor series and averaging term by term. 


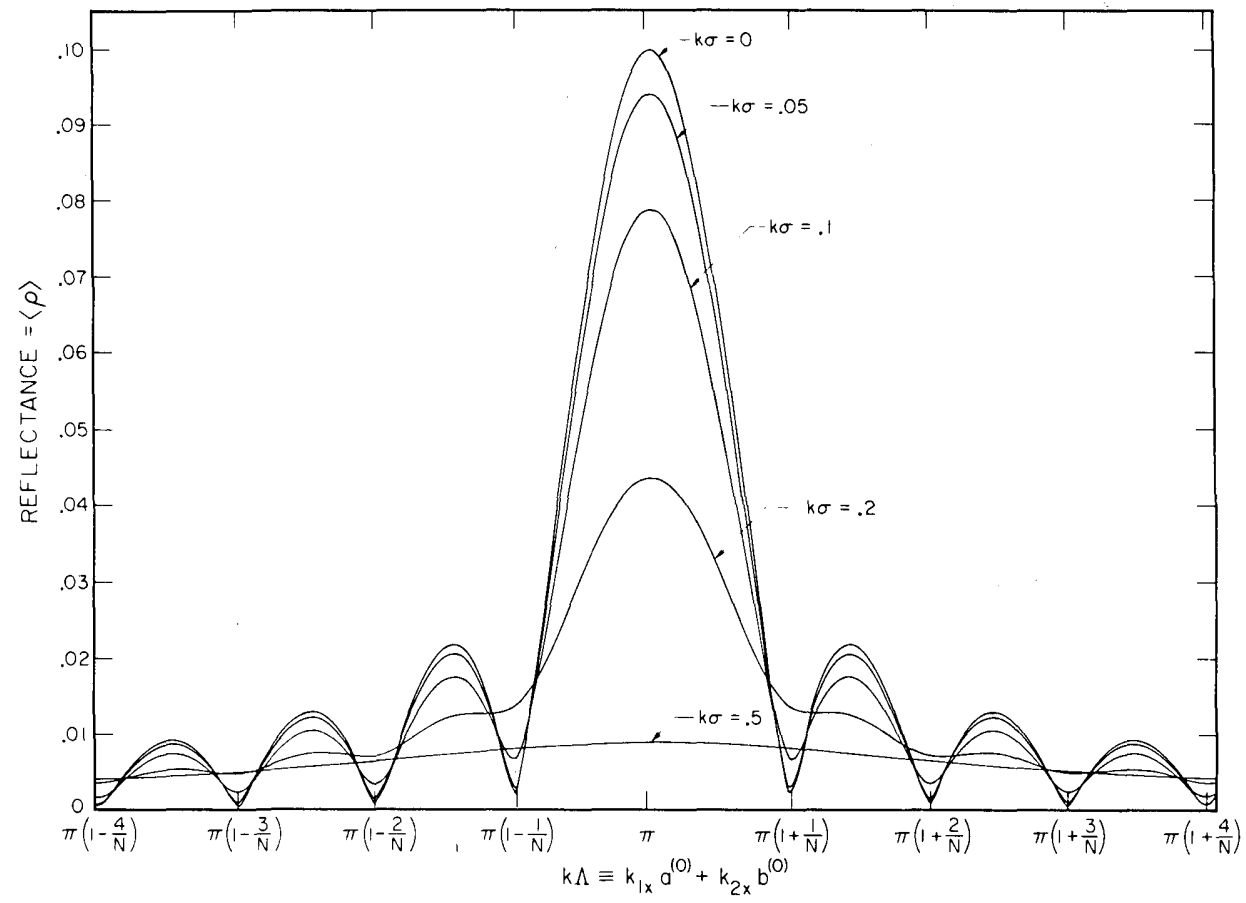

FIG. 2. Average reflectance as a function of layer standard deviation and shift from center of the band gap, indicating the broadening and lowering of the response curve, for the case of 25 cells.

\section{A typical term in (1) is}

$$
\begin{gathered}
\exp \left\{2 i\left[k_{1 x}\left(a_{1}+\cdots+a_{p}\right)+k_{2 x}\left(b_{1}+\cdots+b_{p}\right)\right]\right\} \\
=\exp \left\{2 i p\left[k_{1 x} a^{(0)}+k_{2 x} b^{(0)}\right]\right\} \\
\times \exp \left\{2 i\left[k_{1 x}\left(u_{1}+\cdots+u_{p}\right)+k_{2 x}\left(v_{1}+\cdots+v_{p}\right)\right]\right\} .
\end{gathered}
$$

The ensemble average of this term is

$$
e^{2 i p\left[k_{1 x} a^{(0)}+k_{2 x} b^{(0)}\right]} e^{-2\left[k_{1 x}^{2} p \sigma_{a}^{2}+k_{2 x}^{2} p \sigma b\right]}=e^{2 i p k \Lambda} e^{-2 p k^{2} \sigma^{2}},
$$

where

$$
\begin{aligned}
& k^{2} \sigma^{2} \equiv k_{1 x}^{2} \sigma_{a}^{2}+k_{2 x}^{2} \sigma_{b}^{2}, \\
& k \Lambda \equiv k_{1 x} a^{(0)}+k_{2 x} b^{(0)} .
\end{aligned}
$$

We thus find that the expectation value of $\rho$ is

$$
\begin{aligned}
& \langle\rho\rangle=\left[r_{1} /\left(1-e^{2 i k \Lambda} e^{\left.-2 k^{2} \sigma^{2}\right)}\right]\right. \\
& \times\left[1-e^{2 i(N+1) k \Lambda} e^{-2(N+1) k^{2} \sigma^{2}}-e^{2 i k_{2 x} b^{(0)}}\right. \\
& \left.\times e^{-2 k_{2 x}^{2} \sigma_{b}^{2}}\left(1-e^{2 i N k \Lambda} e^{\left.-2 N k^{2} \sigma^{2}\right)}\right)\right] .
\end{aligned}
$$

The magnitude of this quantity is plotted in Fig. 2 for the case $N=25$ and $r_{1}=1.96 \times 10^{-3}$. We have taken $k_{2 x}^{2} \sigma_{b}^{2}=$ $k_{1 x}^{2} \sigma_{a}^{2}=1 / 2 k^{2} \sigma^{2}$ and $\rho=0.1$ for $\sigma=0$ at the center of the band gap, as well as $k_{2 x} b^{(0)}=k_{1 x} a^{(0)}$. It can be seen in Fig. 2 that the nonzero value of $\sigma$ has the effect of broadening the response as well as lowering it, as expected. Figure 3 is a plot of $\langle\rho\rangle$ as a function of $k \sigma$ for various values of $N$ at the condition $k \Lambda=\pi$, indicating the increasing sensitivity of $\langle\rho\rangle$ on $\sigma$ for large $N$ values. The parameters of each structure have been chosen to give a $10 \%$ reflectance for a perfect reflector. In the limit of $N k^{2} \sigma^{2} \ll 1, N \gg 1$, and $e^{2 i k_{2 x} b^{(0)}}=-1$, expression (4) reduces to

$$
\begin{aligned}
\langle\rho\rangle= & (2 N+1) r_{1}\left(1-N k^{2} \sigma^{2}\right) \\
= & r_{0}\left(1-N k^{2} \sigma^{2}\right) .
\end{aligned}
$$

It is interesting to compare this expression to the wellknown Debye-Waller factor for x-ray diffraction from a crystal at a finite temperature for which ${ }^{13}$

$$
\langle\rho\rangle=r_{0} e^{-G^{2} \sigma^{2} / 6} \approx r_{0}\left[1-\left(G^{2} / 6\right) \sigma^{2}\right],
$$

where $G=k_{\text {in }}-k_{\text {out }}, \sigma$ is the standard deviation in atomic position due to lattice vibration. Note that (5) does not depend on $N$, the number of atomic layers, while the correction factor in (4a) does depend on $N$.

The difference between (4a) and (5) can be reconciled. We consider a structure for which the thickness of each layer can be controlled precisely, but for which the surface of each layer is not perfect but is rough and uneven, then the reflection from the entire structure is reduced by a term similar to (5), that is, independent of $N$. This can be seen as follows. The reflection from a rough surface is given ${ }^{14}$ by $\rho_{0} \exp \left[\left(-8 \pi \sigma^{2} / \lambda^{2}\right)\right.$ $\cos ^{2} \theta$, where $\rho_{0}$ is the reflection from a smooth surface, $\lambda$ is the wavelength of light reflected, $\sigma$ is the standard deviation of the surface from its average, and $\theta$ is the angle of incidence. Again, taking the case where $k_{2 x} b^{(0)}=k_{1 x} a^{(0)}=\pi / 2$ we find

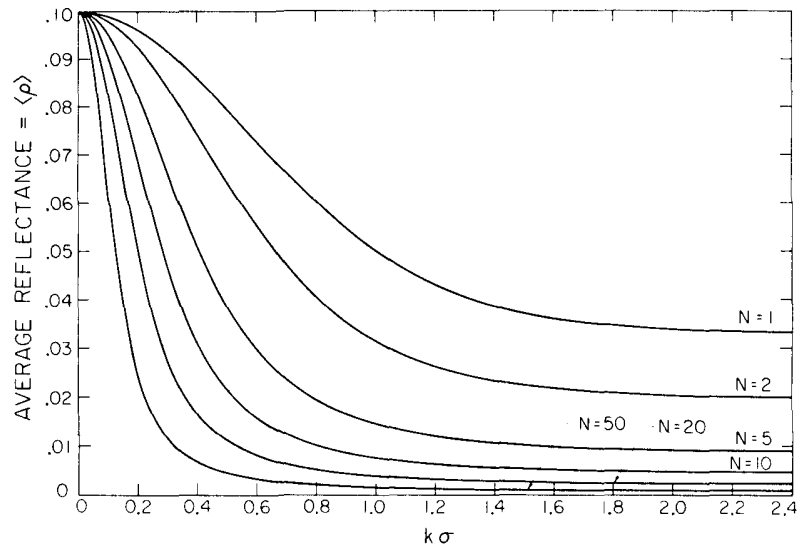

FIG. 3. Average amplitude reflectance in low-reflectance limit as a function of cell standard deviation and the number of cells. Note that the parameters of each structure have been chosen to give a $10 \%$ reflectance for a perfect reflector. 


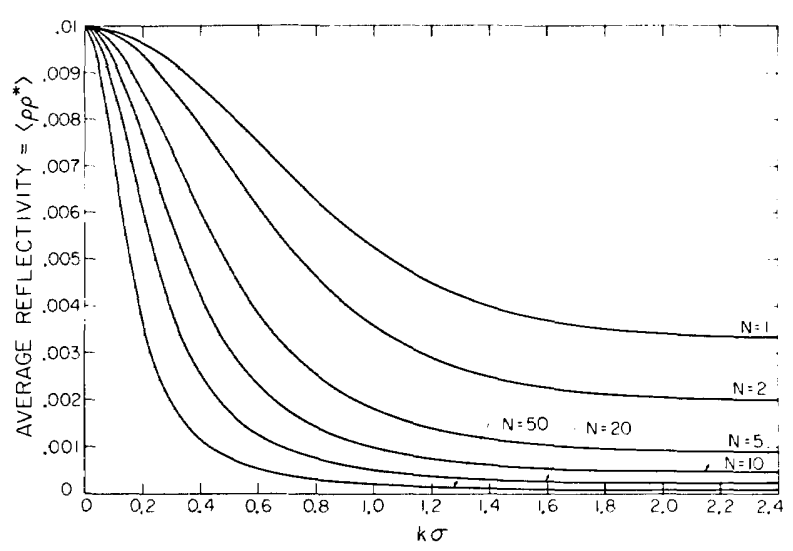

FIG. 4. Average intensity reflectivity in low-reflectance limit as a function of cell standard deviation and the number of cells. Note how, as $\mathrm{k} \sigma$ becomes large the asymptotic reflectance becomes $0.01 /(2 N+1)$.

that the reflection from each surface of a Bragg reflector is reduced by the factor $\exp \left[-(\pi / 2) \sigma_{r_{i}}^{2}\right]$, where $\sigma_{r_{i}}$ is the relative standard deviation, $\sigma_{r_{a}}^{2}=\sigma_{a} / a^{(0)}$ for layers of index $n_{1}$, and $\sigma_{r_{b}}=\sigma_{b} / b^{(0)}$ for layers of index $n_{2}$.

Thus for a structure of many layers, we have

$$
\langle\rho\rangle=1 / 2 r_{0}\left[e^{-(\pi / 2) \sigma_{r a}^{2}-(\pi / 2) \sigma_{r_{b}}^{2}}\right] \approx r_{0}\left[1-(\pi / 4)\left(\sigma_{r_{a}}^{2}+\sigma_{r b}^{2}\right)\right],
$$

which is independent of $N$.

The quantity $\left\langle\rho \rho^{*}\right\rangle$ can be calculated in a manner similar to $\langle\rho\rangle$. If we take, for simplicity, the case $k \Lambda=\pi$, we arrive at the rather complicated expression:

$$
\begin{aligned}
& \left\langle\rho \rho^{*}\right\rangle=\left|r_{1}\right|^{2}\left[\left(N+1+\frac{2 e^{-2 N k^{2} \sigma^{2}}}{\left(1-e^{\left.2 k^{2} \sigma^{2}\right)^{2}}\right.}\left[1-(N+1) e^{2 k^{2} \sigma^{2} N}\right.\right.\right. \\
& \left.\left.+N e^{2(N+1) k^{2} \sigma^{2}}\right]\right)+\left(N+\frac{2 e^{-2(N-1) k^{2} \sigma^{2}}}{\left(1-e^{\left.2 k^{2} \sigma^{2}\right)^{2}}\right.}\right. \\
& \left.\times\left[1-N e^{2 k^{2} \sigma^{2}(N-1)}+(N-1) e^{2 N k^{2} \sigma^{2}}\right]\right) \\
& -\left(2 \cos \left(2 k_{2 x} b_{1}^{(0)}\right)\left(e^{-2 k_{2 x}^{2} \sigma_{b}^{2}}+\mathrm{e}^{-2 k_{1 x}^{2} \sigma_{a}^{2}}\right)\right. \\
& \times \frac{e^{-2(N-1) k^{2} \sigma^{2}}}{\left(1-e^{2 k^{2} \sigma^{2}}\right)^{2}}\left[1-N e^{2(N-1) k^{2} \sigma^{2}}\right. \\
& \left.\left.\left.+(N-1) e^{2 N k^{2} \sigma^{2}}\right]+N\right)\right]
\end{aligned}
$$

In the limit of $N k^{2} \sigma^{2} \ll 1,(7)$ reduces to

$$
\begin{aligned}
&\left\langle\rho \rho^{*}\right\rangle=\left|r_{1}\right|^{2}\{[ 2 N^{2}+2 N+1 \\
&\left.-2\left(N^{2}+N\right) \cos \left(2 k_{2 x} b^{(0)}\right)\right]-k^{2} \sigma^{2}\left[\left(\frac{4}{3} N^{3}+2 N^{2}+\frac{2}{3} N\right)\right. \\
&\left.\left.-2\left(\frac{2}{3} N^{3}+N^{2}+\frac{N}{3}\right) \cos \left(2 k_{2 x} b^{(0)}\right)\right]\right\} .
\end{aligned}
$$

Equation (7) is plotted in Fig. 4 for various values of $N$ under the same conditions as Fig. 3. Although Eq. (7) is quite complicated, it reduces to $\left[(2 N+1) r_{1}\right]^{2}$ for $k \sigma \rightarrow 0$ and to $(2 N$ $+1)\left(r_{1}^{2}\right)$ as $k \sigma \rightarrow \infty$. This is to be expected, since as $\sigma \rightarrow 0$, the reflections from each dielectric interface are correlated and thus the amplitudes add. For large $k \sigma$ values, the reflections from each interface are not correlated and the intensities from the $2 N+1$ interfaces add.

\section{COUPLED MODE THEORY}

In many cases of practical interest, we deal with high reflectivities and the methods of the previous section are inappropriate. This problem can be overcome by using the coupled mode theory ${ }^{15,16}$ and the effects of a random statistical variation in layer thickness can be included by using a perturbation scheme similar to Streifer et al., ${ }^{17}$ but carried to higher order.

Consider a periodic structure that extends from $z=-L / 2$ to $z=\mathrm{L} / 2$. A wave propagating in the $z$ direction, $R(z) e^{i \beta z}$, with time dependence $e^{-i \omega t}$, will generate a contradirectional wave $S(z) e^{-i \beta z}$. We take the dielectric constant as varyıng according to $\cos \left[\left(2 \pi z / \Lambda_{0}\right)+\epsilon \phi(z)\right]$, where $\Lambda_{0}$ is the ideal period and $\epsilon \phi(z)$ describes the perturbation or deviation from this ideal period. Although the dielectric constant of a periodic slab guide does not vary sinusoidally, we can decompose the index variation into its Fourier components and allow coherent interaction with the propagating wave and the first Fourier harmonic of the structure.

The coupled mode equations at the Bragg condition $\beta=$ $\pi / \Lambda_{0}$ are

$$
\begin{gathered}
R^{\prime}=i_{\kappa} e^{i \epsilon \phi} S, \\
S^{\prime}=-i \kappa e^{-i \epsilon \phi} R,
\end{gathered}
$$

where the prime denotes derivative with respect to $z$. In order to keep the results fairly simple we will solve the problem at the Bragg condition only. In Eqs. (9) and (10) $K$ is the coupling constant. It is seen from Eq. (10) that

$$
\begin{gathered}
\kappa=\left|\frac{d S / d z}{R}\right|=\left|\frac{\text { a.r. } / \text { u.l. }}{\text { a.i. }}\right| \\
=\frac{r_{1}}{\Lambda_{0} / 2}=\frac{2 N r_{1}}{L}
\end{gathered}
$$

where a.r. is amplitude reflected, u.l. unit length, and a.i. amplitude incident.

The boundary conditions are

$$
\begin{gathered}
R(-1 / 2 L)=1, \\
S(1 / 2 L)=0 .
\end{gathered}
$$

The filter function or reflection coefficient is defined

$$
\rho\left(-\frac{1}{2}\right)=S\left(-\frac{L}{2}\right) / R\left(-\frac{L}{2}\right)=S\left(-\frac{L}{2}\right) \text {. }
$$

Equations (9) and (10) can be combined to give

$$
R^{\prime \prime}-i \epsilon \phi^{\prime} R^{\prime}-\kappa^{2} R=0 .
$$

Next we expand $R$ in a power series in $\epsilon$ :

$$
R=R_{0}(z)+\epsilon R_{1}(z)+\epsilon^{2} R_{2}(z)+\cdots .
$$

When this is substituted in Eq. (13) and powers of $\epsilon$ are equated, we get

$$
\begin{gathered}
R_{0}^{\prime \prime}-\kappa^{2} R_{0}=0, \\
R_{1}^{\prime \prime}-\kappa^{2} R_{1}=i \phi^{\prime} R_{0}^{\prime},
\end{gathered}
$$




$$
\begin{gathered}
R_{2}^{\prime \prime}-\kappa^{2} R_{2}=i \phi^{\prime} R_{1}^{\prime}, \\
R_{n}^{\prime \prime}-\kappa^{2} R_{n}=i \phi^{\prime} R_{n-1}^{\prime}, \quad n \geqslant 1 .
\end{gathered}
$$

These equations, subject to the boundary conditions of Eqs. (11) and (12) are solved in Appendix A, where it is shown

$$
\begin{gathered}
R_{0}=\frac{\cosh [\kappa(1 / 2 L-z)]}{\cosh \kappa L} \\
R_{n}=\frac{1}{\kappa} \int_{-L / 2}^{z} i \phi^{\prime}(\xi) R_{n-1}^{\prime}(\xi) \sinh [\kappa(z-\xi)] d \xi \\
-\frac{i}{\kappa C_{1}} \frac{\sinh [\kappa(1 / 2 L+z)]}{\cosh \kappa L} \\
\times \int_{-L / 2}^{L / 2} \phi^{\prime}(\xi) R_{n-1}^{\prime}(\xi) \cosh [\kappa(1 / 2 L-\xi)] d \xi \quad n=1,2 .
\end{gathered}
$$

The reflection coefficient $\rho(-L / 2)$ is given by

$$
\rho\left(-\frac{L}{2}\right)=\frac{-i}{\kappa} e^{-i \epsilon \phi(-L / 2)} R^{\prime}\left(-\frac{L}{2}\right) .
$$

If we consider an ensemble of these structures each will have a different reflection since $\epsilon \phi^{\prime}(\mathrm{z})$ is a random variable for each structure. In order to proceed we must consider the autocorrelation function of $\phi^{\prime}(\mathrm{z})$, which we will take as

$$
\begin{gathered}
R_{\phi}\left(z_{0}\right) \equiv\left\langle\phi^{\prime}(z) \phi^{\prime}\left(z+z_{0}\right)\right\rangle \\
\equiv \lim _{W \rightarrow \infty} \frac{1}{2 W} \int_{z=-W}^{W} \phi^{\prime}(z) \phi^{\prime}\left(z+z_{0}\right) d z \\
=\sum^{2}\left(1-\frac{\left|z_{0}\right|}{l}\right) \quad \text { for }\left|z_{0}\right| \leq l \\
=0 \quad \text { for }\left|z_{0}\right| \geq l .
\end{gathered}
$$

Expression (19b) is an assumed form for the autocorrelation function.

The quantity $l$ is a correlation length and $\Sigma^{2}=\left\langle\phi^{\prime 2}\right\rangle$ is the standard deviation of the random variable. Also we assume $\left\langle\phi^{\prime}\right\rangle=0$. This will be discussed further in the next section.

Using the results of Appendix B and Appendix C, we arrive at the following results for $\langle\rho(-L / 2)\rangle$ and $\langle\rho(-L / 2)$ $\left.\rho^{*}(-L / 2)\right\rangle$ :

$$
\begin{aligned}
&\left\langle\rho\left(-\frac{L}{2}\right)\right\rangle=i e^{-i \epsilon \phi(-L / 2)}\left[\tanh \kappa L-\frac{\epsilon^{2} \sum^{2} l}{8 \kappa C_{1}^{3}}\right. \\
& \times\left.\left(2 C_{1}^{5}-\frac{1}{4} S_{1} S_{4}-C_{1} C_{2}-C_{1}+\kappa L S_{1}\right)\right] \\
&\left\langle\rho\left(-\frac{L}{2}\right) \rho^{*}\left(-\frac{L}{2}\right)\right\rangle=\tanh ^{2} \kappa L-\frac{\epsilon^{2} \sum^{2} l}{2 \kappa C_{1}^{4}}\left[\frac{\kappa L}{4}-\frac{1}{16} S_{4}\right. \\
&\left.-\frac{S_{1}}{2}\left(C_{1} C_{2}+C_{1}-2 C_{1}^{5}-\kappa L S_{1}+\frac{1}{4} S_{1} S_{4}\right)\right]
\end{aligned}
$$

Where $S_{n} \equiv \sinh \left(n_{\kappa} L\right), C_{n} \equiv \cosh \left(n_{\kappa} L\right)$. In the low-reflection limit (20) and (21) reduce to

$$
\begin{aligned}
& \left\langle\rho\left(-\frac{L}{2}\right)\right\rangle=\mathrm{ie}^{-\mathrm{i} \epsilon \phi(-\mathrm{L} / 2)} \kappa L\left(1-\frac{\epsilon^{2} \sum^{2} L l}{4}\right), \\
& \left\langle\rho\left(-\frac{L}{2}\right) \rho^{*}\left(-\frac{L}{2}\right)\right\rangle=\kappa^{2} L^{2}\left(1-\frac{\epsilon^{2} \sum^{2} L l}{6}\right) .
\end{aligned}
$$

In the high-reflection limit

$$
\begin{aligned}
\left\langle\rho\left(-\frac{L}{2}\right)\right\rangle & =i e^{-i \epsilon \phi(-L / 2)}\left(\tanh \kappa L-\frac{\epsilon^{2} \sum^{2} l}{8 \kappa}\right), \\
\left\langle\rho\left(-\frac{L}{2}\right) \rho^{*}\left(-\frac{L}{2}\right)\right\rangle=\tanh ^{2} \kappa L & \\
& -\frac{\epsilon^{2} \sum^{2} l}{2 \kappa} e^{-2 \kappa L}(2 \kappa L-1) \\
& \approx 1-e^{-2 \kappa L}\left[4+\frac{\epsilon^{2} \sum^{2} l}{\kappa}\left(\kappa L-\frac{1}{2}\right)\right] .
\end{aligned}
$$

CONNECTION BETWEEN $\epsilon^{2} \Sigma^{2}, I$ AND, $a^{(0)}, b^{(0)}$ AND $\sigma^{2}$ OF THE SLAB REFLECTOR CONSIDERED IN THE LOW-REFLECTIVITY LIMIT SECTION

In order to apply the results of the last section which assumed a sinusoidal variation of the index to the case of multilayered mirrors with abrupt index discontinuities, we establish the connection between the parameters used in characterizing these systems.

We start by defining the local period through the relationship

$$
\begin{gathered}
\left.\left.\int^{z} \frac{2 \pi}{\Lambda(z)} d z \equiv \int^{z}\left(\frac{2 \pi}{\Lambda_{0}}-\frac{2 \pi}{\Lambda_{0}^{2}} \delta \Lambda\right) z\right)\right) d z=\frac{2 \pi z}{\Lambda_{0}}+\epsilon \phi(z) \\
\epsilon \phi^{\prime}(z)=\frac{-2 \pi}{\Lambda_{0}^{2}} \delta \Lambda(z)
\end{gathered}
$$

where $\delta \Lambda(z)$ is the local period variation.

Next we take the autocorrelation function of $\delta \Lambda(z)$ to be

$$
\begin{aligned}
R_{\delta \Lambda}\left(z_{0}\right)=\left\langle\delta \Lambda\left(z+z_{0}\right) \delta \Lambda(z)\right\rangle= & S^{2}\left(1-\frac{\left|z_{0}\right|}{l^{\prime}}\right) \quad\left|z_{0}\right| \leq l^{\prime} \\
& =0 \quad \text { otherwise. }
\end{aligned}
$$

From Eq. (27) we obtain

$$
\begin{aligned}
\epsilon^{2}\left\langle\phi^{\prime}(z) \phi^{\prime}\left(z+z_{0}\right)\right\rangle & =\frac{4 \pi^{2}}{\Lambda_{0}^{4}}\left\langle\delta \Lambda(z) \delta \Lambda\left(z+z_{0}\right)\right\rangle, \\
\epsilon^{2} \sum^{2}\left(1-\frac{\left|z_{0}\right|}{l}\right) & =\frac{4 \pi^{2}}{\Lambda_{0}^{4}} S^{2}\left(1-\frac{\left|z_{0}\right|}{l^{\prime}}\right) .
\end{aligned}
$$

From Eq. (29) we see immediately that

$$
\begin{gathered}
\epsilon^{2} \sum^{2}=\left(4 \pi^{2} / \Lambda_{0}^{4}\right) S^{2}, \\
l^{\prime}=l .
\end{gathered}
$$

Next we relate $l$ to $\Lambda_{0} \equiv a^{(0)}+b^{(0)}$ and $S^{2}$ to $\sigma^{2}$.

As shown in Appendix $\mathrm{D}$ the autocorrelation function for a slab reflector is given by

$$
\begin{aligned}
& \left\langle\Delta t(z) \Delta t\left(z+z_{0}\right)\right\rangle=\bar{\sigma}^{2}\left[1-\left(2\left|z_{0}\right| / \Lambda_{0}\right)\right], \\
& \bar{\sigma}^{2}=1 / 2\left(\sigma_{\mathrm{a}}^{2}+\sigma_{b}^{2}\right), \quad \Lambda_{0} / 2 \approx a^{(0)} \approx b^{(0)} .
\end{aligned}
$$

The quantity $\Delta t(z)$ is the deviation in the slab located at $z$ from its ideal thickness of $\Lambda_{0} / 2$. Comparing Eqs. (29) and (32) we see that the correlation length $l$ is equal to the slab thickness $\Lambda_{0} / 2$. In order to find the connection between $\bar{\sigma}^{2}$ and $\Sigma^{2}$ [and thus $S^{2}$ through Eq. (30)], we compare either Eq. 
(22) to Eq. (5) or alternatively Eq. (23) to Eq. (8) (in limit of large $N)$. In either case for the equations to agree, we must take

$$
\begin{gathered}
S^{2}=(2 \bar{\sigma})^{2}, \\
\epsilon^{2} \sum^{2}=\left(4 \pi^{2} / \Lambda_{0}^{4}\right)(2 \bar{\sigma})^{2} .
\end{gathered}
$$

Thus we have related the quantities $\bar{\sigma}^{2}$ and $\Lambda_{0} / 2$ which are assumed known for our slab Bragg reflector to the quantities $\epsilon^{2} \Sigma^{2}$ and $l$ which appear in Eqs. (20)-(25).

Also by comparing Eq. (5) to (22) or Eq. (8) to (23) (for large $N)$ we again find $k=2 N r_{1} / L$.

After using Eq. (34) and $l=\Lambda_{0} / 2$, Eqs. (20) and (21) become

$$
\begin{gathered}
\left\langle\rho\left(-\frac{L}{2}\right)\right\rangle=i e^{-i \epsilon \phi(-L / 2)}\left[\tanh \kappa L-\frac{\bar{\sigma}^{2} \pi^{2}}{\Lambda_{0}^{3} \kappa C_{1}^{3}}\right. \\
\left.\times\left(2 C_{1}^{5}-\frac{1}{4} S_{1} S_{4}-C_{1} C_{2}-C_{1}+\kappa L S_{1}\right)\right] \\
\left\langle\rho\left(-\frac{L}{2}\right) \rho^{*}\left(-\frac{L}{2}\right)\right\rangle=\tanh ^{2} \kappa L-\frac{4 \bar{\sigma}^{2} \pi^{2}}{\Lambda_{0}^{3} \kappa C_{1}^{4}}\left[\frac{\kappa L}{4}-\frac{1}{16}\right. \\
\left.\times S_{4}-\frac{S_{1}}{2}\left(C_{1} C_{2}+C_{1}-2 C_{1}^{5}-\kappa L S_{1}+\frac{1}{4} S_{1} S_{4}\right)\right] \\
=\tanh ^{2} x-\frac{2 \pi^{2} \bar{\sigma}^{2} L}{\Lambda_{0}^{3}} G(x)
\end{gathered}
$$

or

$$
\begin{gathered}
\frac{\left.\left\langle\rho[-(L / 2)] \rho^{*}[-L / 2)\right]\right\rangle-\tanh ^{2} x}{\tanh ^{2} x}=\frac{-2 \pi^{2} \bar{\sigma}^{2} L}{\Lambda_{0}^{3}} \frac{G(x)}{\tanh ^{2} x}, \\
x \equiv \kappa L, \\
G(x) \equiv-\frac{1}{x}\left[\frac{1}{2 C_{1}^{4}}\left(\frac{1}{4} S_{4}-x\right)\right. \\
\left.+\frac{S_{1}}{C_{1}^{4}}\left(C_{1} C_{2}+C_{1}-2 C_{1}^{5}-x S_{1}+\frac{1}{4} S_{1} S_{4}\right)\right] .
\end{gathered}
$$

The function $G(x)$ is plotted in Fig. 5. For small $x, G(x) \approx$ $(2 / 3) x^{2}$, while for large $x, G(x) \approx 2 e^{-2 x}[2-(1 / x)]$.

\section{ALTERNATIVE DERIVATION USING COUPLED MODE THEORY}

If Eqs. (9) and (10) are used directly and a series expansion is used in both $R$ and $S$, we can avoid having to use Eq. (13), which involves $\phi^{\prime}$, and instead work only with the random variable $\phi$. The procedure is outlined below:

$$
\begin{gathered}
R^{\prime}=i_{\kappa} e^{i \epsilon \phi} S, \\
S^{\prime}=-i_{\kappa} e^{-i \epsilon \phi} R, \\
R^{\prime}=i_{\kappa} S+i_{\kappa}\left(e^{i \epsilon \phi}-1\right) S, \\
S^{\prime}=-i_{\kappa} R-i_{\kappa}\left(e^{-i \epsilon \phi}-1\right) R,
\end{gathered}
$$

where

$$
\epsilon \phi(z)=\frac{\omega}{c} n_{1} \sum_{i=1}^{z / \Lambda} u_{i}+\frac{\omega}{c} n_{2} \sum_{i=1}^{z / \Lambda} v_{i}
$$

[see Eq. (2) for definition of $u_{i}$ and $v_{i}$ ] (boundaries of reflector between $z=0$ and $z=\mathrm{L}$ ).

We define the random variable $\chi=i_{\kappa}\left(e^{i \epsilon \phi}-1\right)$, assume it

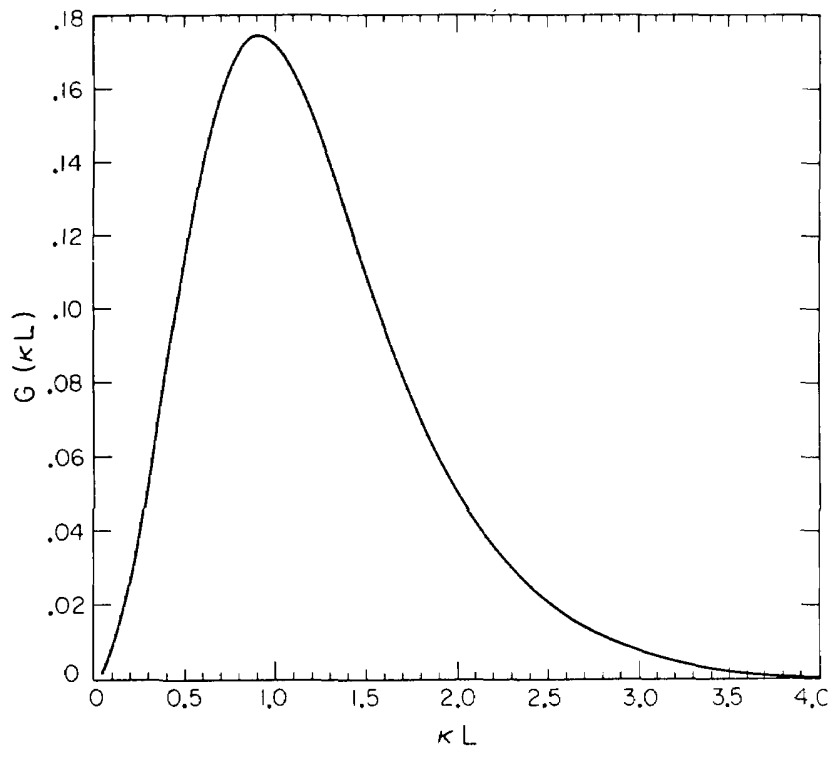

FIG. 5. Plot of the function $G(\kappa L)$.

is small, and expand $R$ and $S$ in a series, with the $n$th term being of order $\chi^{n}$

$$
\begin{aligned}
& R=R_{0}+R_{1}+R_{2}+\cdots, \\
& S=S_{0}+S_{1}+S_{2}+\cdots,
\end{aligned}
$$

with boundary conditions $R_{n}(0)=\delta_{n 0}, S_{n}(\mathrm{~L})=0$. After substituting (42) and (43) into (39) and (40) and collecting terms of order $\chi$, we find

$$
\begin{gathered}
R_{0}^{\prime}=i_{\kappa} S_{0}, \\
S_{0}^{\prime}=-i_{\kappa} R_{0}, \\
R_{n}^{\prime}=i_{\kappa} S_{n}+\chi S_{n-1}, \quad n \geqslant 1 \\
S_{n}^{\prime}=-i \kappa R_{n}+\chi * R_{n-1}, \quad n \geqslant 1 .
\end{gathered}
$$

This system of equations can be solved iteratively for increasing $n$. We will consider only first order and take the region of space containing the reflectors between $z=0$ and $z$ $=\mathrm{L}$ :

$$
\rho \approx \frac{\mathrm{S}_{0}(0)+\mathrm{S}_{1}(0)}{\mathrm{R}_{0}(0)}=\mathrm{S}_{0}(0)+\mathrm{S}_{1}(0)
$$

Solving Eqs. (44) and (45), we find

$$
\begin{gathered}
R_{0}(z)=\frac{\cosh \kappa(L-z)}{C_{1}}, \\
S_{0}(z), \frac{+i \sinh \kappa(L-z)}{C_{1}} .
\end{gathered}
$$

Next we combine Eqs. (46) and (47) to find an equation for $S_{1}$ :

$$
S_{1}^{\prime \prime}-\kappa^{2} S_{1}=-i \kappa \chi S_{0}+\chi^{* \prime} R_{0}+\chi^{*} R_{0}^{\prime} .
$$

Using the boundary conditions on $S_{1}$, integrating by parts to get rid of the derivative of $\chi^{*}$, and using Eqs. (49) and (50) we find

$$
\begin{aligned}
& S_{1}(0)=\frac{i \kappa}{C_{1}^{2}} \int_{0}^{L}\left[\left(e^{-i \epsilon \phi}-1\right) \cosh ^{2} \kappa(L-z)\right. \\
&\left.-\left(e^{i \epsilon \phi}-1\right) \sinh ^{2} \kappa(L-z)\right] d z,
\end{aligned}
$$




$$
\begin{aligned}
\rho \rho^{*}= & \left|S_{0}(0)+S_{1}(0)\right|^{2} \approx\left|S_{0}\right|^{2}+S_{1} S_{0}^{*}+S_{1}^{*} S_{0}, \\
& \Delta r^{2} \equiv \rho \rho^{*}-\left|S_{0}\right|^{2}=S_{0}\left(S_{1}^{*}-S_{1}\right) .
\end{aligned}
$$

After using Eqs. (50) and (52), we arrive at the result

$$
\Delta r^{2}=\frac{-\kappa \tanh \kappa L}{\cosh ^{2} \kappa L} \int_{0}^{L}\left(2-e^{i \epsilon \phi(z)}-e^{-i \epsilon \phi(z)}\right) d z .
$$

(41)

$$
\begin{gathered}
\left\langle\epsilon^{2} \phi^{2}(z)\right\rangle= \\
=\frac{\omega^{2}}{c^{2}} \frac{z}{\Lambda}\left(n_{1}^{2} \sigma_{a}^{2}+n_{2}^{2} \sigma_{b}^{2}\right) \\
=\frac{2 z \Psi}{\Lambda} \\
\Psi \equiv\left(\omega^{2} / 2 c^{2}\right)\left(n_{1}^{2} \sigma_{a}^{2}+n_{2}^{2} \sigma_{b}^{2}\right) \\
\left\langle\Delta r^{2}\right\rangle=\frac{-2 \kappa S_{1}}{C_{1}^{3}} \int_{0}^{L}\left(1-e^{-\Psi(z / \Lambda)}\right) d z \\
=-\frac{2 \kappa S_{1} \Lambda}{C_{1}^{3}}\left(\frac{N \Psi+e^{-N \Psi}-1}{\Psi}\right) .
\end{gathered}
$$

Finally the fractional decrease in average reflectivity is

$$
\frac{\left\langle\Delta r^{2}\right\rangle}{r_{0}^{2}}=\frac{-2 \kappa \Lambda}{\tanh \kappa L \cosh ^{2} \kappa L}\left(\frac{N \Psi+e^{-N \Psi}-1}{\Psi}\right) .
$$

Although this formula is simpler than Eq. (36b), it is not as accurate, as we shall see in the next section. Greater accuracy could be obtained by including higher-order terms in $S$.

\section{COMPUTER RESULTS}

The analytic results of the last section are compared to calculated reflectivity values of a large number of computersimulated stratified media. The multilayer samples were "fabricated" such that the thickness of each layer was a random variable assuming Gaussian distribution about predetermined thicknesses $a_{0}$ and $b_{0}$ of the $n_{1}$ and $n_{2}$ layers, respectively. The same relative standard deviation was used for all layers, i.e., $\sigma_{a} / a^{(0)}=\sigma_{b} / b^{(0)}$. The reflectivity was calculated using the matrix multiplication method. (For a de-

\begin{tabular}{|c|c|c|c|c|c|}
\hline Number of cells & $R_{p}$ & $\langle R\rangle_{\text {Exper }}$ & $\frac{R_{p}-\langle R\rangle_{\text {Exper }}}{R_{p}}$ & $\frac{R_{p}-\langle R\rangle_{\text {theory }} \phi}{R_{p}}$ & $\frac{R_{p}-\langle R\rangle_{\text {Theory }} \phi}{R_{p}}$ \\
\hline 5 & .07743 & .07695 & $6.20 \pm .20 \times 10^{-3}$ & $6.11 \times 10^{-3}$ & $4.67 \times 10^{-3}$ \\
\hline 10 & .26680 & .26420 & $9.75 \pm .19 \times 10^{-3}$ & $9.75 \times 10^{-3}$ & $8.00 \times 10^{-3}$ \\
\hline 15 & .48289 & .47785 & $10.4 \pm .20 \times 10^{-3}$ & $10.6 \times 10^{-3}$ & $9.41 \times 10^{-3}$ \\
\hline 20 & .66500 & .65870 & $9.47 \pm .20 \times 10^{-3}$ & $9.47 \times 10^{-3}$ & $9.22 \times 10^{-3}$ \\
\hline 25 & .79469 & .78865 & $7.60 \pm .15 \times 10^{-3}$ & $7.45 \times 10^{-3}$ & $8.07 \times 10^{-3}$ \\
\hline 30 & .87840 & .87343 & $5.66 \pm .11 \times 10^{-3}$ & $5.49 \times 10^{-3}$ & $6.53 \times 10^{-3}$ \\
\hline 35 & .92942 & .92573 & $3.97 \pm .06 \times 10^{-3}$ & $3.79 \times 10^{-3}$ & $5.01 \times 10^{-3}$ \\
\hline 40 & .95952 & .95695 & $2.68 \pm .04 \times 10^{-3}$ & $2.50 \times 10^{-3}$ & $3.69 \times 10^{-3}$ \\
\hline 45 & .97694 & .97523 & $1.75 \pm .04 \times 10^{-3}$ & $1.68 \times 10^{-3}$ & $2.63 \times 10^{-3}$ \\
\hline 50 & .98691 & .98580 & $1.12 \pm .01 \times 10^{-3}$ & $1.04 \times 10^{-3}$ & $1.83 \times 10^{-3}$ \\
\hline & & & $\sigma_{r}$ & & $\begin{array}{l}n_{1}=3.6 \\
n_{2}=3.4\end{array}$ \\
\hline
\end{tabular}
tailed discussion of the method, the reader is referred to Ref. 2.)

Samples of 50 cells each were prepared this way. The reflectivity was calculated for each sample every 5 cells, giving

TABLE I. Table comparing results of the computer experiment with the two analytic expressions. Note the close agreement between the second order $\phi^{\prime}$ expression and the experiment. $R_{p}=\rho \rho^{*}$ for perfect structure; $\langle R\rangle=\left\langle\rho \rho^{*}\right\rangle ; \sigma_{r}=0.02 ; n_{1}=3.6 ; n_{2}=3.4$.

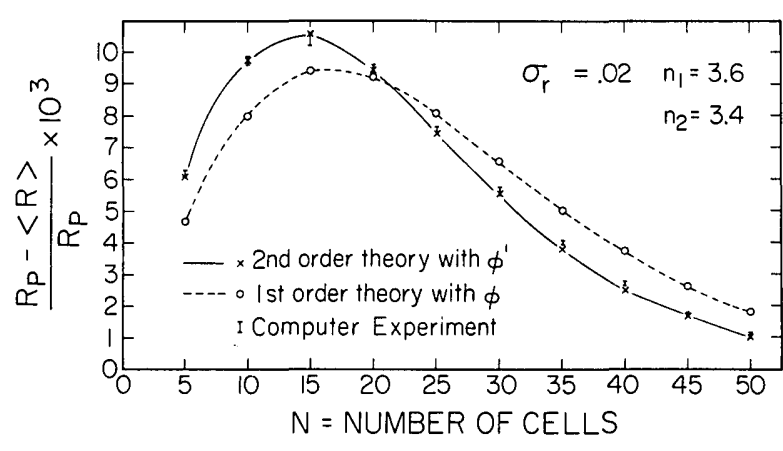

FIG. 6. Plot of the data given in Table I $\left(\sigma_{r}=2 \%\right)$.

reflectivity values of stratified media of $5,10,15,25,30,35$, 40,45 , and 50 cells. There were 1500 such samples. The parameters used in the calculations were $n_{1}=3.6, n_{2}=3.4$, $a^{(0)}=n_{2} \Lambda_{0} /\left(n_{1}+n_{2}\right)=0.4857 \Lambda_{0}, b^{(0)}=n_{1} \Lambda_{0} /\left(n_{1}+n_{2}\right)=$ $0.5143 \Lambda_{0}\left(\Lambda_{0} \equiv a^{(0)}+b^{(0)}\right), \omega=\pi c / 2 n_{1} a^{(0)}$, and normal incidence.

Each sample structure has a different reflection, but all are less than $R_{p} \equiv\left\langle\rho \rho^{*}\right\rangle_{p}$, the reflection from a perfect structure where $\sigma=0$. The average reflection $\langle R\rangle \equiv\left\langle\rho \rho^{*}\right\rangle$ is then determined as well as $\sigma_{\langle R\rangle}=\left(\left\langle R^{2}\right\rangle-\langle R\rangle^{2}\right)^{1 / 2}$, the standard deviation of the reflection. The standard deviation of the computed quantity $\langle R\rangle$ is then determined by $\sigma_{\langle R\rangle} / \sqrt{N}$, where $N$ is the number of structures tested. The value $N=$ 1500 was taken to insure sufficient accuracy in $\langle R\rangle$.

The results of the computer experiment are presented in Table I. For comparison, results are also given for the two analytic theories. The results are plotted in Fig. 6. Values of $\sigma_{r} \equiv \sigma_{a} / a^{(0)}=\sigma_{b} / b^{(0)}=0.02$ were used.

In Fig. 7 are the results for a structure with 10 unit cells and various values of $\sigma_{r}$. It can be seen that there is excellent agreement between the computer results and the second order theory using $\phi^{\prime}$ for small values of $\sigma_{r}$. The first-order theory using $\phi$ also gives good results.

Finally, Figs. 8-13 illustrate the probability distribution function for various reflections. The points were determined by the computer routine, while the solid line represents the theoretical prediction which is described in the next section.

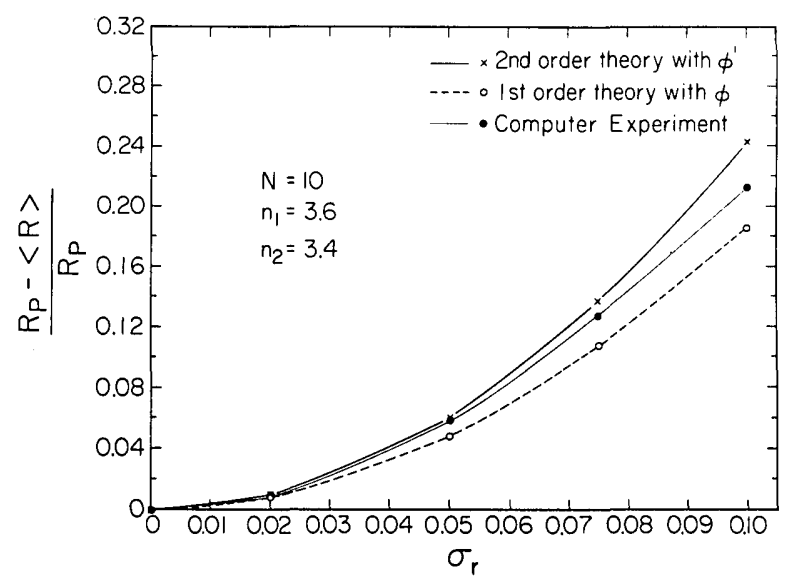

FIG. 7. Comparison of computer experimental results and theory for a 10-cell structure with various values of $\sigma_{r}$. 


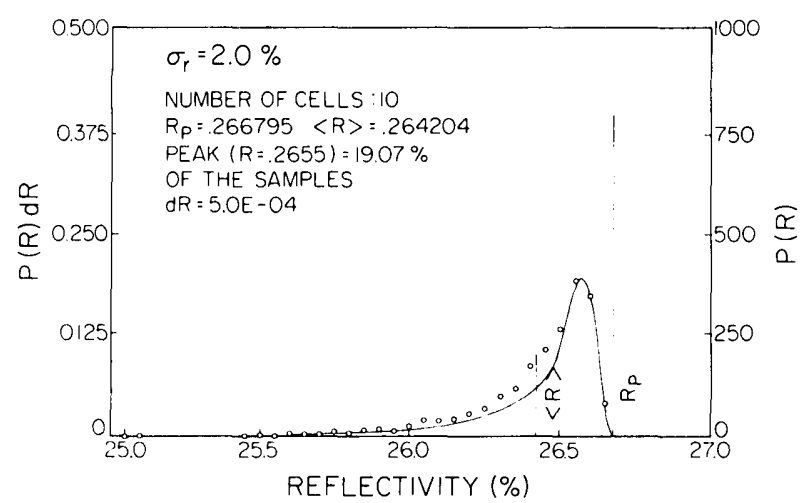

FIG. 8. Experimental distribution of $P(R)$ (circles) as compared to theoretical prediction (solid line) for $\sigma_{r}=2 \%$ for structures with 10 cells. The average reflectivity $\langle R\rangle$ and the reflectivity of a perfect structure $R_{p}$ are indicated by the thin vertical lines.

$P(R) d R$ gives the probability of a structure having reflection between $R$ and $R+d R$. The vertical axis on the right-hand side gives $P(R)$, while the vertical axis on the left-hand side gives $P(R) d R$ with $d R$ specified. Figures $8-10$ give the probability distribution for 10,25 , and 50 cells with relative standard deviation of $2 \%$. Notice how the distribution is broader for 25 cells than for 10 or 50 cells.

In Figs. 11-13 we take a structure of 25 cells and plot the probability distributions for relative standard deviations of $5 \%, 7.5 \%$, and $10 \%$. The same scale is used in these three figures and the broadening of $P(R)$ with standard deviation is readily seen.

\section{A PHENOMENOLOGICAL EXPRESSION FOR $p\left(|\rho|^{2}\right)$}

As seen from Figs. 8-13, the value of $\left\langle\rho \rho^{*}\right\rangle=\langle R\rangle$, while an important parameter of the reflector, does not describe the spread in distribution, or the most likely value of reflectivity. It also does not answer the following important question. If the value of $\sigma$ is allowed to increase, how many structures will no longer satisfy a given required reflectivity? For example, from Fig. 13 we see that although the reflectivity from a perfect structure is almost $80 \%$, a substantial number of reflectors reflect less than $60 \%$. Also, it may be desirable to relax the manufacturing tolerances if this does not lead to a large increase in the number of "bad" mirrors.

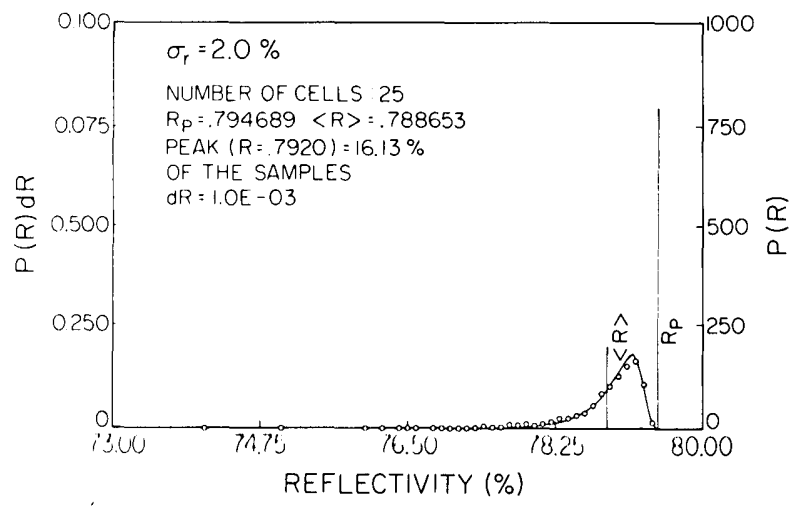

FIG. 9. Same as Fig. 8 , but for structure with 25 cells.

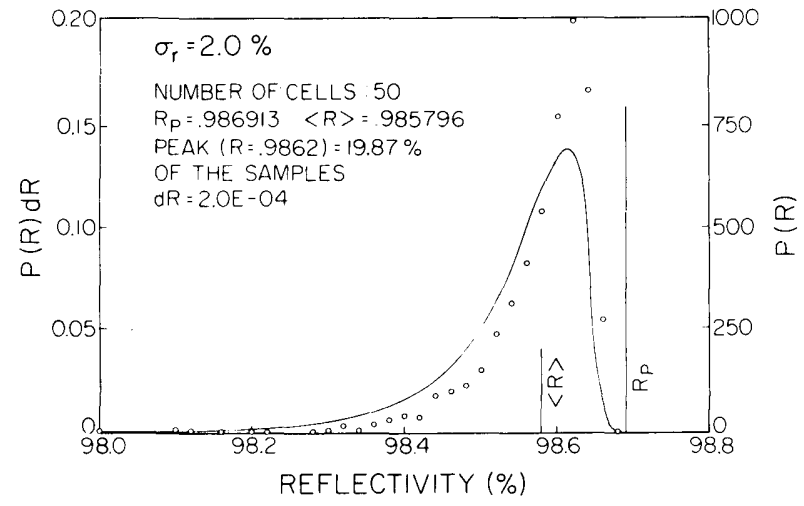

FIG. 10. Same as Fig. 8 , but for structure with 50 cells.

Based on Figs. 8-13 we will fit this data to the function

$$
p(r)=\frac{C^{q-1}}{\Gamma(q-1)} \frac{e^{c /\left(r_{p}-r\right)}}{\left(r_{p}-r\right)^{q}},
$$

where $r=\rho \rho^{*}$ is the reflection, $\Gamma$ is the gamma function, $r_{p}$ is the reflection from a perfect structure, and the parameters $C$ and $q$ are determined from the average value and standard deviation of the distribution function.

It is easily shown that the parameters $C$ and $q$ are related to the average and standard deviation of $p(r)$ through

$$
\begin{gathered}
q=3+\frac{\left(r_{p}-\langle r\rangle\right)^{2}}{\left\langle r^{2}\right\rangle-\langle r\rangle^{2}}, \\
C=\frac{\left(r_{p}-\langle r\rangle\right)\left[\left(r_{p}-\langle r\rangle\right)^{2}+\left(\left\langle r^{2}\right\rangle-\langle r\rangle^{2}\right)\right]}{\left\langle r^{2}\right\rangle-\langle r\rangle^{2}} .
\end{gathered}
$$

From Eq. (55) we have

$$
\begin{gathered}
\langle r\rangle \equiv\left\langle\rho \rho^{*}\right\rangle=r_{p}-\left(\kappa S_{1} / C_{1}^{3}\right)\langle f\rangle, \\
f \equiv \int_{0}^{L}\left(2-e^{i \epsilon \phi(z)}-e^{-i \epsilon \phi(z)}\right) d z, \\
\langle f\rangle=2 \Lambda \frac{N \Psi+e^{-N \Psi}-1}{\Psi} .
\end{gathered}
$$

We need only use Eq. (55) to find $\left\langle r^{2}\right\rangle$. Before proceeding, an important point should be made. In the derivation of (58) we neglected terms of order $S_{n} \alpha\left(e^{i \epsilon \phi}-1\right)^{n} \sim(i \epsilon \phi)^{n}$, where $n>1$. The solution (58) is, however, of order $\phi^{2}$, indicating that terms of $S_{2}$ should have been retained.

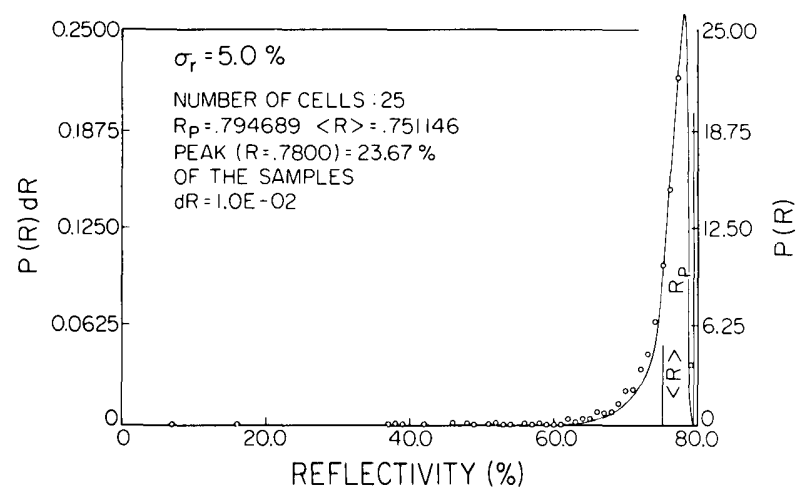

FIG. 11. Experimental distribution of $P(R)$ as compared to theoretical prediction for structures containing 25 cells and have $\sigma_{r}=5 \%$. 


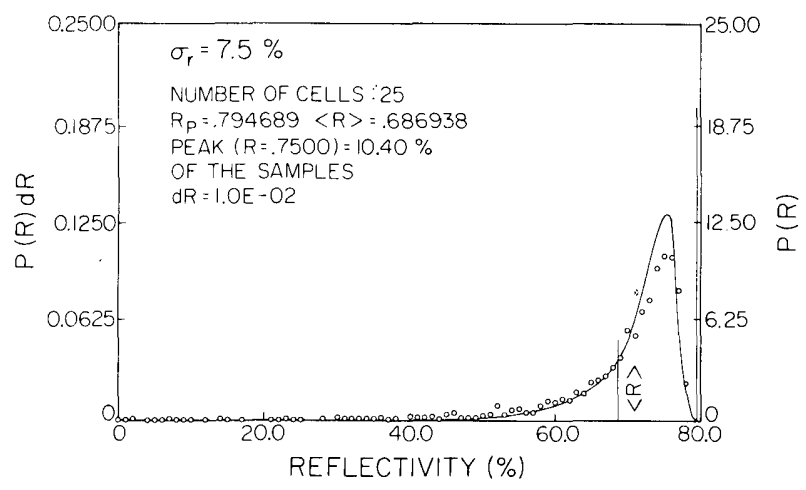

FIG. 12. Same as Fig. 11, but for $\sigma_{r}=7.5 \%$.

Nevertheless the results are in good agreement with experiment as well as the more accurate second-order results, and because of its simplicity expression (55) will be used to compute $\left\langle r^{2}\right\rangle$ as well. Equation (55) gives us

$$
\left\langle r^{2}\right\rangle-\langle r\rangle^{2}=\left(\kappa^{2} S_{1}^{2} / C_{1}^{6}\right)\left(\left\langle f^{2}\right\rangle-\langle f\rangle^{2}\right) .
$$

Using the results of Appendix $\mathrm{E}$ we find

$$
\left(\left\langle r^{2}\right\rangle-\langle r\rangle^{2}\right)^{1 / 2}=\frac{\kappa S_{1}}{C_{1}^{3}} \frac{2}{\sqrt{3}} N L\left(\frac{\pi}{2}\right)^{2}\left(\frac{\bar{\sigma}}{\Lambda / 2}\right)^{2} .
$$

Combining Eqs. (57) and (58), using the definition of $\bar{\sigma}$, and keeping only the lowest-order term in $\bar{\sigma}$, we find

$$
r_{p}-\langle r\rangle=\frac{\kappa S_{1}}{C_{1}^{3}} N L\left(\frac{\pi}{2}\right)^{2}\left(\frac{\bar{\sigma}}{\Lambda / 2}\right)^{2},
$$

or the interesting result

$$
\frac{\left(\left\langle r^{2}\right\rangle-\langle r\rangle^{2}\right)^{1 / 2}}{r_{p}-\langle r\rangle}=\frac{2}{3} \sqrt{3} \approx 1.15
$$

We thus arrive at values for the parameters $q$ and $C$ :

$$
\begin{gathered}
q=\frac{15}{4}, \\
C=\frac{7}{4} \frac{\kappa S_{1}}{C_{1}^{3}} N L\left(\frac{\pi}{2}\right)^{2}\left(\frac{\bar{\sigma}}{\Lambda / 2}\right)^{2},
\end{gathered}
$$

which, when used in (60), give

$$
p(r)=\frac{C^{11 / 4}}{\Gamma(11 / 4)} \frac{e^{C /\left(r_{p}-r\right)}}{\left(r_{p}-r\right)^{15 / 4}}
$$

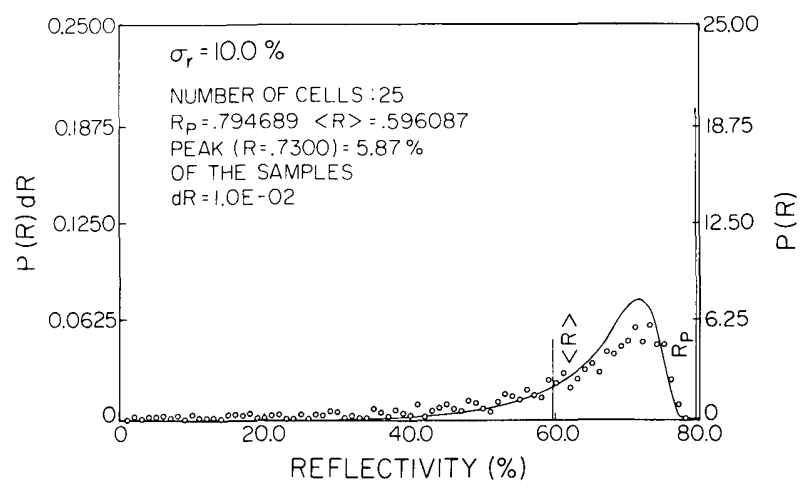

FIG. 13. Same as Fig. 11 , but for $\sigma_{r}=10 \%$. Note the expected broadening of the curve with increasing $\sigma_{r}$.

$$
\Gamma\left(\frac{11}{4}\right)=\left(\frac{7}{4}\right) ! \approx 1.608 \text {. }
$$

The function $p(r)$ is indicated in Figs. 8-13 by the solid line and agrees well with the computer results.

Finally, from Eq. (70) we find that the peak of the function $p(r)$ occurs at the point

$$
r_{\text {peak }}=r_{p}-\frac{C}{q}=r_{p}-\frac{7}{15} \frac{\kappa S_{1}}{C_{1}^{3}} N L\left(\frac{\pi}{2}\right)^{2}\left(\frac{\bar{\sigma}}{\Lambda / 2}\right)^{2},
$$

or

$$
\frac{r_{p}-r_{\text {peak }}}{r_{p}-\langle r\rangle}=\frac{7}{15}
$$

\section{CONCLUSION}

The effect on reflectivity of a statistical variation in the thickness of layers in a Bragg reflector has been studied using the coupled mode equations. Closed-form expressions were obtained for the reduction in reflectivity, which agreed with a computer experiment. These expressions are accurate for small values of $\sigma$, which is typical for most cases. A phenomenological expression for the reflectivity distribution function $p(r)$ was presented which also agreed well with the experiment. Results for arbitrarily large $\sigma$ values were obtained for low-reflectivity reflectors.

\section{APPENDIX A}

In this appendix we solve

$$
R_{0}^{\prime \prime}-\kappa^{2} R_{0}=0
$$

and

$$
R_{n}^{\prime \prime}-\kappa^{2} R_{n}=i \phi^{\prime} R_{n-1}^{\prime}, \quad n=1,2
$$

subject to

$$
R(-1 / 2 L)=1
$$

and

$$
S(1 / 2 L)=0
$$

where

$$
R=R_{0}+\epsilon R_{1}+\epsilon^{2} R_{2}
$$

and

$$
S=-i e^{-i \epsilon \phi}\left(R^{\prime} / \kappa\right)
$$

The procedure for determining $R_{0}$ is straightforward and the solution is

$$
R_{0}(z)=\frac{\cosh \{\kappa[(L / 2)-z]\}}{\cosh (\kappa L)} .
$$

The boundary conditions on $R^{\prime}$ are, from (A 4) and (A 6) $R^{\prime}(L / 2)=0$.

Since these must hold for all values of $\epsilon$ we have

$$
R_{1}\left(-\frac{L}{2}\right)=R_{2}\left(-\frac{L}{2}\right)=R_{1}^{\prime}\left(\frac{L}{2}\right)=R_{2}^{\prime}\left(\frac{L}{2}\right)=0 \text {. }
$$

A homogeneous solution to (A 2) is given by

$$
B_{n} \sinh \{\kappa[(L / 2)+z]\},
$$


where $B_{n}$ is a constant to be determined. The particular solution is given by

$$
\frac{1}{\kappa} \int_{-L / 2}^{z} i \phi^{\prime}(\eta) R_{n-1}^{\prime}(\eta) \sinh [\kappa(z-\eta)] d \eta .
$$

This can be confirmed by differentiating (A 9) and substituting in (A 2).

The total solution is thus

$$
\begin{aligned}
R_{n}(z)=B_{n} \sinh [ & {\left[\left(\frac{L}{2}+z\right)\right] } \\
& \left.+\frac{1}{\kappa} \int_{-L / 2}^{z} i \phi^{\prime}(\eta) R_{n-1}^{\prime}(\eta) \sinh \kappa(z-\eta)\right] d \eta .
\end{aligned}
$$

The boundary solution at $z=-L / 2$ is also satisfied and $B_{n}$ is determined through the boundary condition $R_{n}^{\prime}(L / 2)=0$. The result is given by Eq. (17).

\section{APPENDIX B}

We wish to solve for the expectation value of $\rho(-L / 2)=$ $\langle\rho(-L / 2)\rangle$ given that $\left\langle\phi^{\prime}(z) \phi^{\prime}\left(z+z_{0}\right)\right\rangle=\Sigma^{2}\left[1-\left(\left|z_{0}\right| / l\right)\right]$ and using Eqs. 16-18:

$$
\begin{aligned}
\left\langle R^{\prime}\left(-\frac{L}{2}\right)\right\rangle & =\left\langle R_{0}^{\prime}\left(-\frac{L}{2}\right)\right\rangle \\
+\epsilon & \left\langle R_{1}^{\prime}\left(-\frac{L}{2}\right)\right\rangle+\epsilon^{2}\left\langle R_{2}^{\prime}\left(-\frac{L}{2}\right)\right\rangle .
\end{aligned}
$$

After differentiation we find from Eq. (17)

$$
\begin{array}{r}
\left\langle R_{1}^{\prime}\left(-\frac{L}{2}\right)\right\rangle=\frac{-i}{C_{1}} \int_{-L / 2}^{L / 2}\left\langle\phi^{\prime}(\eta)\right\rangle R_{0}^{\prime}(\eta) \cosh \left[\kappa\left(\frac{L}{2}-\eta\right)\right] \\
d \eta=0 \quad \text { since }\langle\phi(\eta)\rangle=0 \quad \text { (B 2) }
\end{array}
$$

Equation (18) also gives

$$
R_{2}^{\prime}\left(-\frac{L}{2}\right)=\frac{-i}{C_{1}} \int_{-L / 2}^{L / 2} \phi^{\prime}(\xi) R_{1}^{\prime}(\xi) \cosh \left[\kappa\left(\frac{L}{2}-\xi\right)\right] d \xi .
$$

We now use Eq. (17) to express $R_{1}^{\prime}$ in terms of $R_{0}$ and $\phi^{\prime}$. The result is

$$
\begin{aligned}
& \left\langle R_{2}^{\prime}\left(-\frac{L}{2}\right)\right\rangle=\frac{-\kappa}{C_{1}^{2}} \int_{\xi=-L / 2}^{L / 2} \int_{\eta=-L / 2}^{\xi}\left\langle\phi^{\prime}(\xi) \phi^{\prime}(\eta)\right\rangle \cosh \left[\kappa\left(\frac{L}{2}-\xi\right)\right] \cosh [\kappa(\xi-\eta)] \sinh \left[\kappa\left(\frac{L}{2}-\eta\right)\right] d \xi d \eta \\
& +\frac{\kappa}{C_{1}^{3}} \int_{\xi=-L / 2}^{L / 2} \int_{\eta=-L / 2}^{L / 2}\left\langle\phi^{\prime}(\xi) \phi^{\prime}(\eta)\right\rangle \cosh \left[\kappa\left(\frac{L}{2}-\xi\right)\right] \cosh \left[\kappa\left(\frac{L}{2}+\xi\right)\right] \cosh \left[\kappa\left(\frac{L}{2}-\eta\right)\right] \sinh \left[\kappa\left(\frac{L}{2}-\eta\right)\right] d \xi d \eta .
\end{aligned}
$$

From Eq. 19 we have $R_{\phi}(\xi-\eta)=\left\langle\phi^{\prime}(\xi) \phi^{\prime}(\eta)\right\rangle=\Sigma^{2}[1-$ $(|\xi-\eta| / l)]$.

The integration is quite involved unless we make the approximation

$$
R_{\phi}(\xi-\eta) \approx l \sum^{2} \delta(\xi-\eta),
$$

where $\delta(x)$ is the Dirac delta function.

This is a quite reasonable approximation and is good whenever $k l \ll 1$. After using

$$
\int_{a}^{b} \delta(x) f(x) d x=f(0) \text { for } a<0<b
$$

and

$$
\int_{0}^{b} \delta(x) f(x) d x=1 / 2 f(0) \text { for } \quad b>0
$$

we find

$$
\begin{aligned}
\left\langle R_{2}^{\prime}\left(-\frac{L}{2}\right)\right\rangle=\frac{\sum^{2} l}{8 C_{1}^{3}}\left(-C_{1} C_{2}-C_{1}\right. & \\
& \left.\quad+2 C_{1}^{5}+{ }^{\kappa} L S_{1}-\frac{1}{4} S_{1} S_{4}\right) .
\end{aligned}
$$

When this is combined with (B 1$)$ and Eq. (18), we arrive at Eq. (20).

\section{APPENDIX C}

The expectation value of $\rho(-L / 2) \rho^{*}(L / 2)$ is calculated in a fashion similar to $\langle\rho(-L / 2)\rangle$ :

$$
\begin{aligned}
\rho\left(-\frac{L}{2}\right)=\frac{-i}{\kappa} e^{-i \epsilon \phi(-L / 2)} & {\left[R_{0}^{\prime}\left(-\frac{1}{2} L\right)\right.} \\
+\epsilon R_{1}^{\prime} & \left.\left(-\frac{1}{2} L\right)+\epsilon^{2} R_{2}^{\prime}\left(-\frac{1}{2} L\right)\right]
\end{aligned}
$$

$$
\begin{aligned}
\left\langle\rho\left(-\frac{1}{2} L\right) \rho^{*}\left(-\frac{1}{2} L\right)\right\rangle & =\frac{1}{\kappa^{2}}\left(R_{0}^{\prime} R_{0}^{\prime *}+\epsilon^{2}\left\langle R_{1}^{\prime} R_{1}^{\prime *}\right\rangle\right. \\
& \left.+\epsilon^{2}\left\langle R_{0}^{\prime} R_{2}^{\prime *}+R_{0}^{\prime *} R_{2}^{\prime}\right\rangle\right)_{z}=-L / 2 .
\end{aligned}
$$

All terms of order $\epsilon$ do not contribute since $\left\langle\phi^{\prime}\right\rangle=0$ (see Appendix B):

$$
\left\langle R_{0}^{\prime} R_{2}^{\prime *}+R_{0}^{\prime *} R_{2}^{\prime}\right\rangle=R_{0}^{\prime}\left\langle R_{2}^{\prime *}\right\rangle+R_{0}^{\prime *}\left\langle R_{2}^{\prime}\right\rangle .
$$

$\left\langle R_{2}^{\prime}\right\rangle$, the expectation value of $R_{2}^{\prime}$ has been calculated in Appendix B. Thus we need only determine $\left\langle R_{1}^{\prime} R_{1}^{\prime *}\right\rangle$

$$
\begin{gathered}
\left\langle R_{1}^{\prime}\left(-\frac{L}{2}\right) R_{1}^{\prime *}\left(-\frac{L}{2}\right)\right\rangle=\frac{\kappa^{2}}{C_{1}^{4}} \int_{-L / 2}^{L / 2} \int_{-L / 2}^{L / 2}\left\langle\phi^{\prime}(\eta) \phi^{\prime}(\xi)\right\rangle \\
\quad \times \sinh \left[\kappa\left(\frac{L}{2}-\eta\right)\right] \sinh \left[\kappa\left(\frac{L}{2}-\xi\right)\right] \\
\times \cosh \left[\kappa\left(\frac{L}{2}-\eta\right)\right] \cosh \left[\kappa\left(\frac{L}{2}-\xi\right)\right] d \eta d \xi . \quad(\mathrm{C})
\end{gathered}
$$

Using (B 5), this reduces to

$$
\left\langle R_{1}^{\prime}\left(-\frac{L}{2}\right) R_{1}^{\prime *}\left(-\frac{L}{2}\right)\right\rangle=\frac{\kappa \sum^{2} l}{C_{1}^{4}}\left(\frac{1}{32} S_{4}-\frac{1}{8} \kappa\right) \cdot
$$
21.

\section{APPENDIX D}

In this appendix we solve

$$
\begin{aligned}
& R_{t}\left(z_{0}\right)=\left\langle\Delta t(z) \Delta t\left(z+z_{0}\right)\right\rangle \\
= & \lim _{W \rightarrow \infty} \frac{1}{2 W} \int_{-W}^{W} \Delta t(z) \Delta t\left(z+z_{0}\right) d z .
\end{aligned}
$$


Equation (D 1) can be interpreted as

$$
\left\langle\Delta t(z) \Delta t\left(z+z_{0}\right)\right\rangle=\sigma_{a}^{2} P_{n_{1}}\left(z_{0}\right)+\sigma_{b}^{2} P_{n_{2}}\left(z_{0}\right),
$$

where $P_{n_{i}}\left(z_{0}\right)$ is the probability that two points separated by a distance $z_{0}$ will both be in the same cell of index $n_{i}$.

From Ref. 18 we have

$$
\begin{gathered}
P_{n_{1}}=\frac{a_{0}}{\Lambda_{0}}\left(1-\frac{\left|z_{0}\right|}{a_{0}}\right) \text { for }\left|z_{0}\right|<a_{0}, \\
=0 \quad \text { otherwise, } \\
P_{n_{2}}=\frac{\mathrm{b}_{0}}{\Lambda_{0}}\left(1-\frac{\left|\mathrm{z}_{0}\right|}{\mathrm{b}_{0}}\right) \text { for }\left|z_{0}\right|<b_{0}, \\
=0 \quad \text { otherwise, }
\end{gathered}
$$

where we have assumed $\sigma_{a} / a_{0}, \sigma_{b} / b_{0} \ll 1$.

If we now combine Eqs. (D 2) and (D 3) and take $a_{0} \sim b_{0} \sim$ $\Lambda_{0} / 2$, we obtain

$$
\begin{array}{ccc}
\left\langle\Delta t(z) \Delta t\left(z+z_{0}\right)\right\rangle=\bar{\sigma}^{2} & \left(1-\frac{\left|z_{0}\right|}{\Lambda_{0} / 2}\right) & \left|z_{0}\right|<\Lambda_{0} / 2 \\
=0 & \text { otherwise. }
\end{array}
$$

\section{APPENDIX E}

In this appendix we find an expression for $\left\langle\mathrm{f}^{2}\right\rangle-\langle\mathrm{f}\rangle,{ }^{2}$ where

$$
\begin{gathered}
f \equiv \int_{0}^{L}\left(2-e^{i \epsilon \phi(z)}-e^{-i \epsilon \phi(z)}\right) d z, \\
\epsilon \phi(z)=\frac{\omega}{c} n_{1} \sum_{i=1}^{z / \Lambda} u_{i}+\frac{\omega}{c} n_{2} \sum_{i=1}^{z / \Lambda} v_{i} .
\end{gathered}
$$

The variables $u_{i}$ and $v_{i}$ are Gaussian distributed as described in the main body of this paper:

$$
\begin{aligned}
& \left\langle f^{2}\right\rangle=\left\langle\int _ { 0 } ^ { L } \int _ { 0 } ^ { L } ( 2 - e ^ { i \epsilon \phi ( z ) } - e ^ { - i \epsilon \phi ( z ) } ) \left( 2-e^{i \epsilon \phi\left(z^{\prime}\right)}\right.\right. \\
& \left.\left.-e^{-i \epsilon \phi\left(z^{\prime}\right)}\right) d z d z^{\prime}\right\rangle=4 L^{2}-8 L \int_{0}^{L}\left\langle e^{-i \epsilon \phi(z)}\right\rangle d z \\
& +2 \int_{0}^{L} \int_{0}^{L}\left\langle e^{i \epsilon\left[\phi(z)+\phi\left(z^{\prime}\right)\right]}\right\rangle d z d z^{\prime} \\
& +2 \int_{0}^{L} \int_{0}^{L}\left\langle e^{i \epsilon\left[\phi(z)-\phi\left(z^{\prime}\right)\right]}\right\rangle d z d z^{\prime} .
\end{aligned}
$$

In arriving at (E 3) we used the fact that $\left\langle e^{i \epsilon\left[\phi(z)+\phi\left(z^{\prime}\right)\right]}\right\rangle=$ $\left\langle e^{-i \epsilon\left[\phi(z)+\phi\left(z^{\prime}\right)\right]}\right\rangle$, etc.

We now examine the second, third, and fourth terms on the right side of (E 3$)$ :

$$
\begin{array}{r}
-8 L \int_{0}^{L}\left\langle e^{-i \epsilon \phi(z)}\right\rangle d z=-8 L \int_{0}^{L} e^{\left(\epsilon^{2} / 2\right)\left\langle\phi^{2}(z)\right\rangle} d z \\
=-8 L \int_{0}^{L} e^{(\Psi / \Lambda) z} d z=\frac{8 L^{2}}{N \Psi}\left(e^{-N \Psi}-1\right) .
\end{array}
$$

We now examine

$$
2 \int_{0}^{L} \int_{0}^{L}\left\langle e^{i \epsilon\left[\phi(z)+\phi\left(z^{\prime}\right)\right]}\right\rangle d z d z^{\prime}
$$

$\phi(z)$ and $\phi\left(z^{\prime}\right)$ are not independent. Over the region of integration where $z^{\prime}>z$ we can write $\phi(z)+\phi\left(z^{\prime}\right)=2 \phi(z)+\Omega\left(z^{\prime}\right.$ $-z$ ), where $\phi$ and $\Omega$ are independent and $\Omega(x)$ has the same probability distribution function as $\phi(x)$. Thus

$$
\begin{aligned}
\left\langle e^{i \epsilon\left[\phi(z)+\phi\left(z^{\prime}\right)\right]}\right\rangle & =\left\langle e^{2 i \epsilon \phi(z)}\right\rangle\left\langle e^{i \epsilon \Omega\left(z^{\prime}-z\right)}\right\rangle \\
& =e^{-2 \epsilon^{2}\left(\phi^{2}(z)\right\rangle} e^{-\left(\epsilon^{2} / 2\right)\left\langle\Omega^{2}\left(z^{\prime}-z\right)\right\rangle} \\
& =e^{-4 z \Psi / \Lambda} e^{-(\Psi / \Lambda)\left(z^{\prime}-z\right)},
\end{aligned}
$$

with a symmetric expression for $z>z^{\prime}$. After integrating this third term we find

$$
\begin{aligned}
2 \int_{0}^{L} \int_{0}^{L} & \left\langle e^{i \epsilon\left[\phi(z)+\phi\left(z^{\prime}\right)\right]}\right\rangle d z d z^{\prime} \\
& =\frac{4}{3} \frac{L^{2}}{N^{2} \Psi^{2}}\left(\frac{3}{4}-e^{-N \Psi}+\frac{1}{4} e^{-4 N \Psi}\right) .
\end{aligned}
$$

In a similar manner we find

$2 \int_{0}^{L} \int_{0}^{L}\left\langle e^{i \epsilon\left[\phi(z)-\phi\left(z^{\prime}\right)\right]}\right\rangle d z d z^{\prime}$

$$
\begin{aligned}
&=4 \int_{z=0}^{L} \int_{z^{\prime}=0}^{z} e^{-(\psi / \Lambda)\left(z-z^{\prime}\right)} d z d z^{\prime} \\
&=\frac{4 L^{2}}{N \Psi}\left(1+\frac{1}{N \Psi}\left(e^{-N \Psi}-1\right)\right) .
\end{aligned}
$$

After combining our results and expanding all expressions in a power series in $\Psi$, retaining terms up to order $\Psi^{2}$ we arrive at the desired result:

$$
\left\langle f^{2}\right\rangle-\langle f\rangle^{2}=\frac{4}{3}(N L \Psi)^{2} \simeq \frac{4}{3} N^{2} L^{2}\left(\frac{\pi}{2}\right)^{4}\left(\frac{\bar{\sigma}}{\Lambda / 2}\right)^{4} .
$$

* Research supported by the NSF (Optical Communication Program) and the Air Force Office of Scientific Research.

†Fannie and John Hertz Foundation Doctoral Fellow.

${ }^{1}$ M. Born and E. Wolf, Principles of Optics (MacMillan, New York, 1964).

${ }^{2}$ P. Yeh, A. Yariv and C. Hong, J. Opt. Soc. Am. 67, 423 (1977).

${ }^{3}$ A. Ashkin and A. Yariv, Bell Labs. Tech. Memo MM-61-124-46 (13 November 1961) (unpublished).

${ }^{4}$ N. Bloembergen and A. J. Sievers, Appl. Phys. Lett. 17, 483 (1970).

${ }^{5}$ C. L. Tang and P. P. Bey, IEEE J. Quantum Electron. QE-9, 9 (1973).

6S. M. Rytov, Zh. Eksp. Teor. Fiz. 29, 605 (1955) [Sov. Phys.-JETP 2, 466 (1956)].

7J. P. Vander Ziel, M. Illegems, and R. M. Mikulyak, Appl. Phys. (to be published).

${ }^{8}$ Private communication with Wess Icenogle of Spectra Physics, Mountain View, Calif.

${ }^{9}$ Private communication with Steve Silver of OCLI, Santa Rosa, Calif.

${ }^{10} \mathrm{~K}$. O. Hill, Appl. Opt. 13, 1853 (1974).

${ }^{11}$ A. Katzir, A. C. Livanos, J. B. Shellan, and A. Yariv, IEEE J. Quantum Electron. QE-13, 296 (1977).

${ }^{12}$ C. Hong, J. B. Shellan, A. C. Livanos, A. Yariv, and A. Katzir, Appl Phys. Lett. 31, 276 (1977).

${ }^{13} \mathrm{C}$. Kittel, Introduction to Solid State Physics (Wiley, New York, 1971), p. 85

${ }^{14}$ Petr Beckmann, The Scattering of Electromagnetic Waves From Rough Surfaces (MacMillan, New York, 1963), p. 81.

${ }^{15}$ H. Kogelnik, Bell. Syst. Tech. J. 55, 109-126 (1976).

${ }^{16}$ A. Yariv, IEEE J. Quantum Electron. QE-9, 919 (1973).

${ }^{17}$ W. Streifer, D. R. Scifres, and R. D. Burnham, J. Opt. Soc. Am. 66, 1359 (1976)

${ }^{18} \mathrm{H}$. W. Harman, Principles of the Statistical Theory of Communication, (McGraw-Hill, New York, 1963), p. 75. 